\title{
Identifying customer need patterns for customization and personalization
}

\author{
Keywords \\ Customization, Product attributes, \\ Customer requirements
}

\begin{abstract}
The importance of incorporating customer preferences into product specifications for successful customized product design has been well recognized. An approach based on the identification of customer need patterns is proposed in this paper for better understanding of customer preferences and accordingly to enhance the product definition for customization and personalization. A tree-structured classification algorithm is formulated for the identification of customer need patterns. Accordingly, a twophase methodology is developed for effective product definition. A case study of power supply products is reported to illustrate the feasibility and potential of the proposed approach.
\end{abstract}

\section{Received November 2000 Revised October 2001 Accepted January 2002}

This research was partially supported by the Hong Kong Government Research Grant Council (HKUST 797/96E and HKUST 6220/99E). The authors would like to express their sincere thanks to Dr M. Eugene Merchant, Professors Stephen C.-Y. Lu, Num P. Suh, Gunnar Sohlenius and Martin Helander for their valuable advice. Dr W.K. Lo and Mr Xuanzhong Liu at Artesyn Technologies Asia-Pacific Ltd are acknowledged for their continuous support.

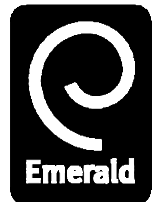

Integrated Manufacturing Systems

14/5 [2003] 387-396

(C) MCB UP Limited

[ISSN 0957-6061]

[DOI 10.1108/09576060310477799]

\author{
Xuehong Du \\ Artesyn Technologies Asia-Pacific Ltd, Hong Kong \\ Jianxin Jiao \\ School of Mechanical and Production Engineering, \\ Nanyang Technological University, Singapore \\ Mitchell M. Tseng \\ Department of Industrial Engineering and Engineering Management, \\ The Hong Kong University of Science and Technology, Hong Kong
}

\section{Introduction}

Enterprises in all branches of industry are being forced to react to the growing individualization of demand, yet, at the same time, increasing competitive pressure dictates that costs must also continue to decrease (The Economist, 2000). To meet this challenge, the economic landscape of the 1990s has been subject to fundamental changes from a manufacturing viewpoint - from mass production to mass customization. Mass customization and personalization aim at providing goods and services that serve individual customers' personal needs with near mass production efficiency (Tseng and Jiao, 1996). It has rapidly gained broad attention by businesses of all branches of industry (The Economist, 2001).

Mass customization and the corresponding concept of personalization have already been discussed in the literature for more than a decade, whereas practical implementation of the principles of mass customization in businesses can be found only in recent years. Academic research and development of the theoretical and managerial aspects of mass customization and personalization are increasing rapidly. While an Internet search of the term mass customization got about 350 results in 1995, nowadays there are almost 70,000 hits. According to a recent literature search, there are more than 2,300 articles published about the topic since the term was formally coined in 1993, about 60 per cent of them within last two years.

The essence of customization and personalization is to provide only and exactly what each customer wants at the right time (Pine and Gilmore, 1999). Von Hippel (1998) stresses the importance of the customer's involvement in designing products for mass customization, since the customer has the very best understanding of his/her own needs, and can relay the information to the manufacturer. Peppers et al. (1999) also emphasize the understanding and categorization of customers as necessary requirements in order for product/service providers to be able to customize their offerings. To avoid the sacrifice of customer goodwill and maintain high customer satisfaction, one of the key issues lies in how to understand customers better, that is, to explore how the customers interact with the product/service providers. Therefore, it is necessary to investigate customer preferences in order to have a better support for the business model of customization and personalization.

Moreover, in terms of product development, traditional mass-produced product providers offer take-all or leave-it options for the customers, whereas customization and personalization provide unique products to cater to individual customer preferences. A popular way of product customization and personalization is by configuration design, where customers can choose different components and assemble them together to form a product. Product families and product platforms have been well recognized for this purpose (Anderson, 1997). The rationale is to provide a generic umbrella to capture and utilize commonality within which each new product instantiated, so as to anchor future designs to a common product line structure (Du et al., 2001). The very first stage of product family design is product definition, a process of translating the voice of customers into product specifications. The purpose is to translate subjective customer needs into objective specifications from which engineers can start to design products (Suh, 1990). Timely, complete and accurate product 
Xuehong Du, Jianxin Jiao and

Mitchell M. Tseng

Identifying customer need

patterns for customization and

personalization

Integrated Manufacturing

Systems

14/5 [2003] 387-396 definition is one of the premises for manufacturers to meet diverse customer needs individually and responsively in today's competitive global market (Pugh, 1991). As observed by Tseng and Jiao (1998), difficulties in communication between customers and designers result in complicated interrelationships between customer needs and product specifications. Some problems may occur, including:

- long lead time due to back and forth interactions between designers and customers with marketing personnel acting as an agent;

- an exponential increase of uncontrolled variety due to the fact that both customers and marketing personnel lack design expertise; and

- consequently high costs and long lead times.

As a result, it is necessary to enhance the product definition for customization and personalization, that is, how to transfer customer preferences to product specifications precisely and rapidly.

Towards this end, this paper proposes an approach based on identification and utilization of customer need patterns for better understanding of customer preferences and subsequently enhancing the product definition for customization and personalization. In the next section, the background leading to this research is reviewed including related work in customer preferences and product definition. In section 3 , an algorithm is presented for the identification of customer need patterns. Based on customer need patterns, a two-phase methodology is developed for effective product definition in section 4 . A case study of power suppliers is reported in section 5 . Finally, the paper is concluded in section 6 .

\section{Background review}

\subsection{Customer preference}

Research in understanding customer preferences, known as consumer behavior study, has been the subject of investigation in the psychological marketing area for a few decades. Dominant work in traditional consumer behavior study has geared toward explaining how consumers behave with the intention to predict aggregate trends. In a scenario of customization and personalization, however, the focus has to be shifted from passive to proactive analysis. It is necessary to understand individual consumers from the designers' side, as well as from the customers' side to provide guidance for consumers to find what they want and to help the customers to explore their preferences through informed choices. This is particularly true for those capital products with sophisticated technical contents such as power suppliers.

Marketing research has pointed out three major challenges for the understanding of customer preferences, involving the product, customer and environment aspects. The product aspect mainly refers to the context-dependence of choice. It means that there are many situational aspects that could change product preferences over different times or different situations. The customer aspect refers to different customer characteristics that could change preferences, for example mood, emotion or impulsive feeling. The environmental aspect refers to those aspects outside the customer, for example influence of family or social environment that could change product preferences. In customization and personalization, the understanding of consumer preferences becomes complicated since it has to be able to capture individual data for each customer.

In addition, there are two streams of disciplines in answering the challenge to understand customer preferences in customization and personalization. The first one is to capture customer preferences through data mining and profiling methods. Data mining and profiling are able to collect demographic and customer preference data, and to extract customer behavior from their preferences. With the support of the advanced Web technology, product providers are able to collect a large sample of such customer preference data. The other way to understand customer preferences in customization and personalization is by extending marketing theories to the customization and personalization situation. This effort mainly involves empirical research, similar to what marketing researchers have been doing for the last 30 years. Huffman and Kahn (1998) investigate consumer behavior when presented with high variety selection tasks. They found that the process could be playful when the customers were informed of product varieties and were able to learn while gathering information. But it could also be frustrating when customers were confused by the amount of product variants - a chaotic case like mass confusion.

The first stream targets the result of customization and personalization, where data are extracted from customer preference and customer demographic data. The other one emphasizes the process of customization 
Xuehong Du, Jianxin Jiao and

Mitchell M. Tseng

Identifying customer need

patterns for customization and

personalization

Integrated Manufacturing

Systems

14/5 [2003] 387-396 and personalization, such as the decision-making process when customizing products. It is a challenging task to investigate customer preferences associated with customizing products with consideration of both the process and the result aspects of customization and personalization.

\subsection{Product definition}

Based on the axiomatic design theory (Suh, 1990), the design world consists of four distinctive domains, i.e. the customer, functional, physical, and process domains. It involves a zigzagging mapping process between domains with creative conceptualization. Each domain is characterized by a characteristic vector, i.e. CNs (customer needs), FRs (functional requirements), DPs (design parameters), and PVs (process variables), respectively. As shown in Figure 1, product definition falls into the first two domains. It begins with customer needs (CNs) and ends with a whole set of product specifications in the form of FRs. Defining FRs by capturing, analyzing, understanding, and projecting CNs is the task of this stage (Tseng and Jiao, 1998).

In terms of product realization for customization and personalization, a company's capability is represented as product offerings and can be perceived from three different perspectives: the customer, design and manufacturing views. These frontend views can be synchronized by product family design at the backend (Tseng and Jiao, 1996). A product family consists of a base product together with those derived products, thus represented by a center vector for the base product with certain ranges for variation. Product family design is supported by an appropriate product platform - a set of subsystems and interfaces that form a common structure from which a stream of derived products can be developed and produced (Mayer and Lehnerd, 1997). To leverage reusability, investment continuity, and responsiveness to changing $\mathrm{CNs}$, the

\section{Figure 1}

Product definition within the framework of axiomatic design

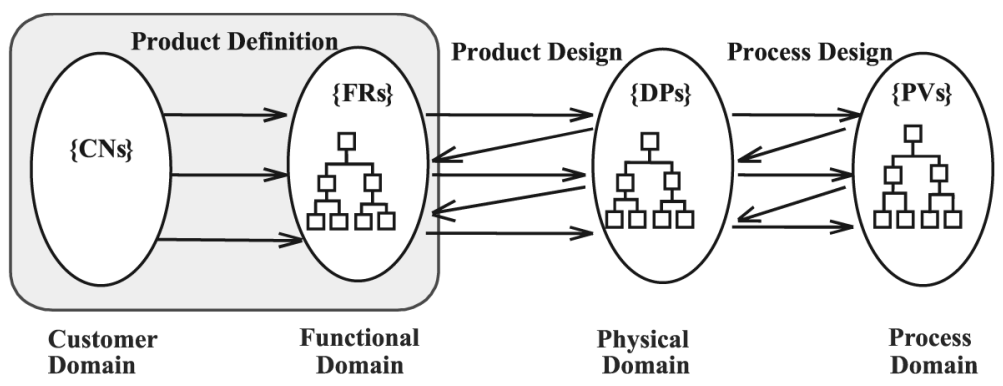

company's capabilities in design and manufacturing should be organized and represented at the backend. Such kind of backend capabilities can be reflected in product specifications. As a whole, the frontend efforts of marketing aim at inducing customer needs to the company's core competency, that is, to increase the convergence of the company's capability to the markets (Tseng and Du, 1998).

\subsubsection{Terminology}

Customer needs. Customers are usually referred to as the end-users of a product. Their needs can be expressed using a vector of customer need variables (CNVs). These variables may be either numerical (i.e. continuous) or categorical (i.e. discrete). Usually they can be classified into certain groups or levels. For high-end products, like TV sets or capital products, this assumption is reasonable, especially when a customer is committed to buying a product rather than only considering a product concept while answering a questionnaire in marketing research.

Though, in general, customer needs are diverse, there exist some patterns that can be characterized by certain determinant variables (Hutt and Thomas, 1998). These eminent variables become the enablers underlying diverse customer needs. Product differentiation manifests itself through different levels of CNVs, from which customers may choose. As noted by Anderson (1997), in a diversified market, customer needs should be mapped to a product family instead of a single product.

Functional requirements. A product specification is described by a set of FRs. They are derived from the requirements of a host of customers, not only end-users, but also internal customers, such as corporate requirements, regulatory requirements, technical requirements, and so on (Morris and Stauffer, 1994). The end-user's requirements, characterized by a set of CNVs, can be regarded as a subset of FRs and less specific than the FRs. In other words, FRs can be regarded as transformed from CNVs with enrichment of engineering considerations. For example, a "good picture" is a customer need for a TV set. It will be interpreted as "high resolution" (e.g. 1,024 lines) as a FR in the product specification. Customers would not specify a power supply when they express their needs for a TV set, but when developing the product specification some parameters relevant to the power supply must be specified (as FRs). Without these additional FRs beyond $\mathrm{CNs}$ themselves, the design of a TV set cannot be initiated. 
Xuehong Du, Jianxin Jiao and

Mitchell M. Tseng

Identifying customer need

patterns for customization and

personalization

Integrated Manufacturing

Systems

14/5 [2003] 387-396
Therefore, FRs are different from CNs in that they must be complete and objective in terms of engineering. Their values are the actual values engineers will use to design products. This fact allows us to analyze FRs using some mathematical tools and to formulate FR templates to facilitate the systematic product definition (Tseng and Jiao, 1998). Behind each FR template is a supporting product platform or product family.

Customer choice. Customer choice of a product depends on explicit requirements, implicit requirements, available options and the latent requirements implied by the product. The underlying relationships of customers' specified requirements and their choice of products are very complicated, putting aside the complexity of product specifications. Therefore, to identify and represent these relationships, inductive learning is more appropriate. If a tree-structured classification technique is adopted, each path from the root of the tree to a terminal node will represent a $\mathrm{CN}$ pattern (Currim et al., 1988).

\subsubsection{FR template}

Tseng and Jiao (1998) recognize the rationale of FR templates with respect to requirement management for product definition. FR templates are formulated to assist design engineers to define product specifications and present them in an organized and systematic manner. By analyzing existing products and historical data, the templates open opportunities for incorporating expert experience into new product design and enhancing the ability to explore and utilize underlying domain knowledge effectively. The formulation of FR templates includes steps depicted below.

FR topology. Depending on specific domain knowledge, the FR topology refers to the terminological product specification variables and the interrelationships among them. Its formulation involves three steps. First, position the company's product offerings with regard to its strength and farm out unprofitable products. Then, define a set of aggregate specification variables based on the existing product portfolio and refine it according to product strategies. FR topology defines the generic features to describe the whole spectrum of product offerings of a company. It provides a systematic organization of product specification to define engineering requirements for a given product.

FR instantiation. Checking with specific product attributes, the desired values (instances) and importance level for each attribute, the specifications of existing products can be mapped into various instances to represent specific products. Through such mapping, useful historical data and domain knowledge are incorporated into and represented by FR instances.

FR template construction. Within each product family there are several products represented by FR instances. Using the fuzzy C-means (FCM) clustering analysis technique (Zimmermann, 1987), similar products in terms of their desired values for specific variables comprise a cluster. Each cluster is characterized by a representative center vector, which is subsequently used as the base value for planned product variants. The variation range of a base value is usually determined according to the variation of specific instances within a cluster of the products. The final FR templates, center values and variation ranges, are constructed by further refining the initial templates in terms of business considerations, like product migration, technological trends, and market competition.

\section{Identification of customer need patterns}

Our previous work (Tseng and Jiao, 1998) on FR patterns emphasizes the functional domain only. This research moves on to the frontend, i.e. considering both the customer and functional domains. Based on analysis of historical data, customer need $(\mathrm{CN})$ patterns can be identified and used to build up the mapping relationships between $\mathrm{CN}$ patterns and FR templates. Accordingly, they are applied to the development of product specifications for a given set of CNs through instantiation. It is expected to help customers make informed choices, elicit CNs and introduce them to the company's capabilities, and accelerate product definition. Table I gives a comparison of $\mathrm{CN}$ patterns and FR templates.

The goal of identifying CN patterns is to construct a rule set in order to predict which FR template is going to be used to define a product. The FR template retrieved should be the closest to meeting a given set of CNs. Essentially this can be regarded as a classification problem.

Classification methods such as clustering analysis and multi-dimensional scaling (MDS) are compensatory models and thus not suitable for purchasing decision making. How to determine weighting of each variable from the company's documents is also a challenge. The tree-structured classification algorithm is adopted in this research owing to its strength in describing decision-making 
Xuehong Du, Jianxin Jiao and
Mitchell M. Tseng

Identifying customer need

patterns for customization and

personalization

Integrated Manufacturing

Systems

14/5 [2003] 387-396

\section{Table I}

CN patterns vs. FR templates

\begin{tabular}{lll}
\hline & Customer need pattern & Functional requirement template \\
\hline Perspective & Customer & Design engineer \\
Origination & Customer requirement repository & Product repository \\
Primary expertise & Marketing & Design \\
Variable & Continuous and categorical & Applicable value \\
Terminology & Customer's & Engineer's \\
Representation & A path from the root to a terminal node in & Center vector plus variation range based on FR \\
& CN classification tree & topology \\
Relationship & Tree structured classification and mapping &
\end{tabular}

processes, as well as being powerful enough to handle data that may be numerical or categorical and may possess complicated interrelationship (Currim et al., 1988; Gunter, 1998). There are three components associated with this algorithm: the training set, the splitting rule, and the stopping rule, as depicted below.

\subsection{Training set formulation}

Customer need variables (CNVs) are usually identified using Pareto analysis or factor analysis. Based on the distribution of each CNV, or the levels of the company's offerings, or other scaling methods (Churchill, 1995), the values of each CNV can be transformed into categorical data, or levels.

Suppose $C N V_{i}$ takes $N_{i}$ levels. For customer profile, $k$ :

$$
\begin{gathered}
C N V^{k} \sim\left(C N V_{1}^{k}, C N V_{2}^{k}, \ldots, C N V_{N}^{k}\right), \\
C N V_{i}^{k} \in\left\{1,2, \ldots, N_{i}\right\},
\end{gathered}
$$

where all $C N V^{k}, k=1,2, \ldots, K$, compose a CN space.

The customer order profile is described as $\left(C N V^{k}, P^{k}\right)$, where $P^{k}$ is the product ordered by customer, $k$. The product can be defined using FR template:

$$
F R T^{k}, F R T^{k} \in\{1,2, \ldots, J\},
$$

where $J$ denotes the total number of FR templates.

Then, we obtain the training set, $L$, defined as:

$$
\begin{gathered}
L \sim\left\{\left(C N V^{1}, F R T^{1}\right),\left(C N V^{2}, F R T^{2}\right),\right. \\
\left.\ldots,\left(C N V^{K}, F R T^{K}\right)\right\} .
\end{gathered}
$$

\subsection{Tree growing algorithm}

The concept learning system (CLS) algorithm (Currim et al., 1988) is modified as follows:

1 For each $C N V_{i}$, define a splitting criterion $C\left(C N V_{i}\right)$, which is a measure of the ability of the particular variable to discriminate which template is to be chosen. Based on the information theory (Shannon, 1948), $C\left(C N V_{i}\right)$ is a measure of the classification power of $C N V_{i}$, or its entropy. Formally, let $f_{l / i}$ be the number of choice options with level $l$ of $C N V_{i}$. Therefore, $C\left(C N V_{i}\right)$ can be defined as:

$$
C\left(C N V_{i}\right)=-\sum_{l=1}^{N_{i}} f_{l / i}
$$

$$
\times\left[\sum_{j=1}^{J} p\left(F R T_{j} \mid l, i\right) \log p\left(F R T_{j} \mid l, i\right)\right],
$$

where $p\left(F R T_{j} \mid l, i\right)$ is the probability (estimated from the sample proportion) of FR template, $j$, being chosen if the $\mathrm{CN}$ variable, $C N V_{i}$, takes on level $l$.

2 Define the CNV with the smallest criterion as the starting variable or primary "root" of the CN classification tree. Partition the data into $N_{i}$ splits. If there is a tie in determining the variable with the lowest criterion value, then select the starting variable at random.

3 With each split, recalibrate the discrimination criterion for remaining variables and define the variable that best discriminates the options as the root of a secondary branch.

4 Continue to apply this heuristic to successive splits of the data until either all instances have been classified or a stopping criterion is reached.

\subsection{Tree structure and size optimization}

The most commonly adopted approach to determine where branching should be halted is based on when the increase in information content about classification provided by the addition of a variable is below some threshold level. Unfortunately, the use of such rules usually does not guarantee an optimal predictive tree. To solve this problem, Breiman (1984) suggested that the decision tree probably can be best estimated by first building a tree without stopping constraints, then successively deleting 
Xuehong Du, Jianxin Jiao and Mitchell M. Tseng

Identifying customer need patterns for customization and personalization

Integrated Manufacturing Systems

14/5 [2003] 387-396 branches until a significant decrease in the model's explanatory ability is encountered.

In this research, we first grow a maximum tree, with each terminal node corresponding to one FR template. Then delete the branches successively. Two scenarios may appear:

1 Each terminal node only has one or a few FR templates. It implies either the structure of the tree is appropriate and predictive, or the branching is too detailed. For the former, we can stop. For the latter, we will try to delete some branches further to see whether the templates falling in the same node can be reformulated as one template or several other templates according to the technical feasibility and economy of scale in connection to product families.

2 Quite a few terminal nodes share the same $F R$ template. It means the structure of the tree is not appropriate and not very predictive. This means we should check the splitting rule and reconstruct the tree. It also possibly implies that some of the CNVs might not be that determinant. It may be necessary to check and re-select the CNVs.

In general, the identification of $\mathrm{CN}$ patterns and the formulation of FR templates interact with each other. Only through joint efforts between marketing personnel and design engineers with a number of iterations can an optimal tree be obtained.

\subsection{An illustrative example}

Suppose there are two CNVs: $A$ and $B$; each taking on three levels, $A_{1}, A_{2}, A_{3}, B_{1}, B_{2}$, and $B_{3}$; with three templates, I, II, and III. There are six customer profiles in the training set, as listed in Table II.

Calculate the splitting criterion:

$$
C(A)=2.60 ; \quad C(B)=1.91 \text {. }
$$

Therefore use $B$ as the primary splitting variable and $A$ as the secondary splitting variable. The tree obtained is shown in Figure 2 .

From the tree shown in Figure 2, some facts can be observed:

\section{Table II}

An illustration of the training set

\begin{tabular}{llll}
\hline Customer & CNV A & CNV B & FR template \\
\hline $\mathbf{1}$ & $\mathrm{A}_{1}$ & $\mathrm{~B}_{1}$ & $\mathrm{I}$ \\
$\mathbf{2}$ & $\mathrm{A}_{1}$ & $\mathrm{~B}_{2}$ & $\mathrm{II}$ \\
$\mathbf{3}$ & $\mathrm{A}_{2}$ & $\mathrm{~B}_{3}$ & $\mathrm{I}$ \\
$\mathbf{4}$ & $\mathrm{A}_{1}$ & $\mathrm{~B}_{2}$ & $\mathrm{II}$ \\
$\mathbf{5}$ & $\mathrm{A}_{3}$ & $\mathrm{~B}_{2}$ & $\mathrm{II}$ \\
$\mathbf{6}$ & $\mathrm{A}_{3}$ & $\mathrm{~B}_{1}$ & $\mathrm{I}$ \\
\hline
\end{tabular}

\section{$\overline{\text { Figure } 2}$}

An illustration of the classification tree

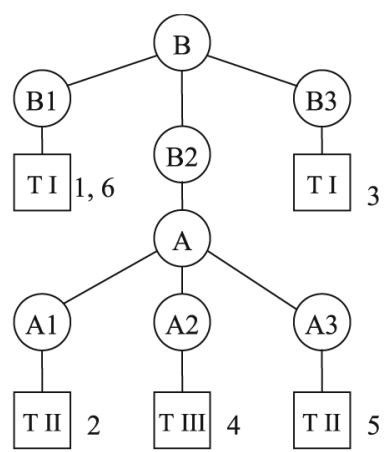

- Customers 1 and 6 share the same pattern. The CN patterns for customers 2,4 , and 5 are more similar to one another than to the others.

- Predictability: once a customer specifies $B_{1}$ or $B_{3}$, we can predict that most likely template I will satisfy the customer needs. It is not necessary to check $A$.

- We can analyze templates II and III to see whether they can be combined or not since from the customers' point of view they are quite similar.

- Contrary to selecting the smallest splitting criterion, if we select $A$ as the root splitting variable, the tree shown in Figure 3 will be obtained. This tree is much more complicated than the one in Figure 2. There are too many FR templates shared by the customers. More customers' needs need to be clarified in order to recommend a suitable one. The predictability is not as good as the previous tree shown in Figure 2. Thus this tree structure is not appropriate, and needs to be modified.

\section{Product definition for customization and personalization}

The challenge of product definition lies in how to assist marketing personnel and

\section{$\overline{\text { Figure } 3}$}

An appropriate tree for the illustrative example

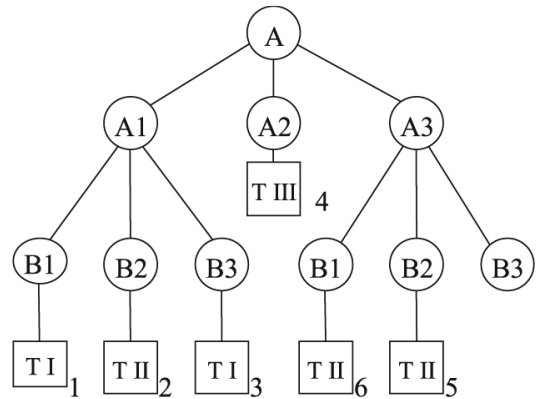


Xuehong Du, Jianxin Jiao and

Mitchell M. Tseng

Identifying customer need

patterns for customization and

personalization

Integrated Manufacturing

Systems

14/5 [2003] 387-396 design engineers work together to elicit CNs, organize and represent product specifications from both engineering and customer perspectives, and how to explicate the relationships between them. The outputs are in the form of a tree-structured classificatory knowledge base. When a customer specifies his/her particular needs for a product, CN patterns are used to retrieve an appropriate FR template, which is used to guide the customer to specify the needs and to derive a complete set of product specifications. Such a CN pattern facilitated product definition will enhance the quality of product specifications and alleviate difficulties associated with tedious, indirect interactions among customers, marketing personnel and designers.

With recognized FR templates and developed product specification generators in Tseng and Jiao (1998), CN patterns and CN classifiers can be accordingly developed. With these two mechanisms, a two-phase methodology can be formulated to assist product definition. As shown in Figure 4, there are two phases involved: the preparation phase and the adoption phase.

\subsection{Preparation phase: cross-function teamwork}

The preparation phase involves cross-function teamwork, in which FR templates are constructed from existing designs and $\mathrm{CN}$ patterns are extracted from customer profiles. The $\mathrm{CN}$ patterns and FR templates are inter-related by constructing a
$\mathrm{CN}$ classification tree. Figure 4 depicts both the FR template formulation process and CN pattern identification process. Tseng and Jiao (1998) discuss the details of the former. The latter is described in section 3 .

Figure 5 gives an overview of the entity relationships involved in the proposed methodology. CNs are first classified into $\mathrm{CN}$ patterns. Each CN pattern is connected to certain FR templates. A FR template is composed of the central values of FRs and their variation ranges. The central value and selected variation are mapped to a detailed product specification supported by product families that represent the company's capability. In this way, the classification tree links CNs to design parameters and fills the gap between the customer domain and the engineering domain.

\subsection{Adoption phase: effective product definition}

The customer is required to input his/her needs. Then these CNs are analyzed to retrieve a relevant $\mathrm{CN}$ pattern. The corresponding FR template will be selected to generate the product specification. The FR template identified through the $\mathrm{CN}$ pattern will meet the particular CNs of the customer.

Once a specific FR template is obtained, the issue becomes whether to use the default base values or to customize the base values within the variation ranges (Tseng and Du, 1998). Such a decision ultimately affects the cost and schedule of product realization. The a priori relationships provide design

\section{$\overline{\text { Figure } 4}$}

A two-phase methodology for effective product definition based on $\mathrm{CN}$ patterns

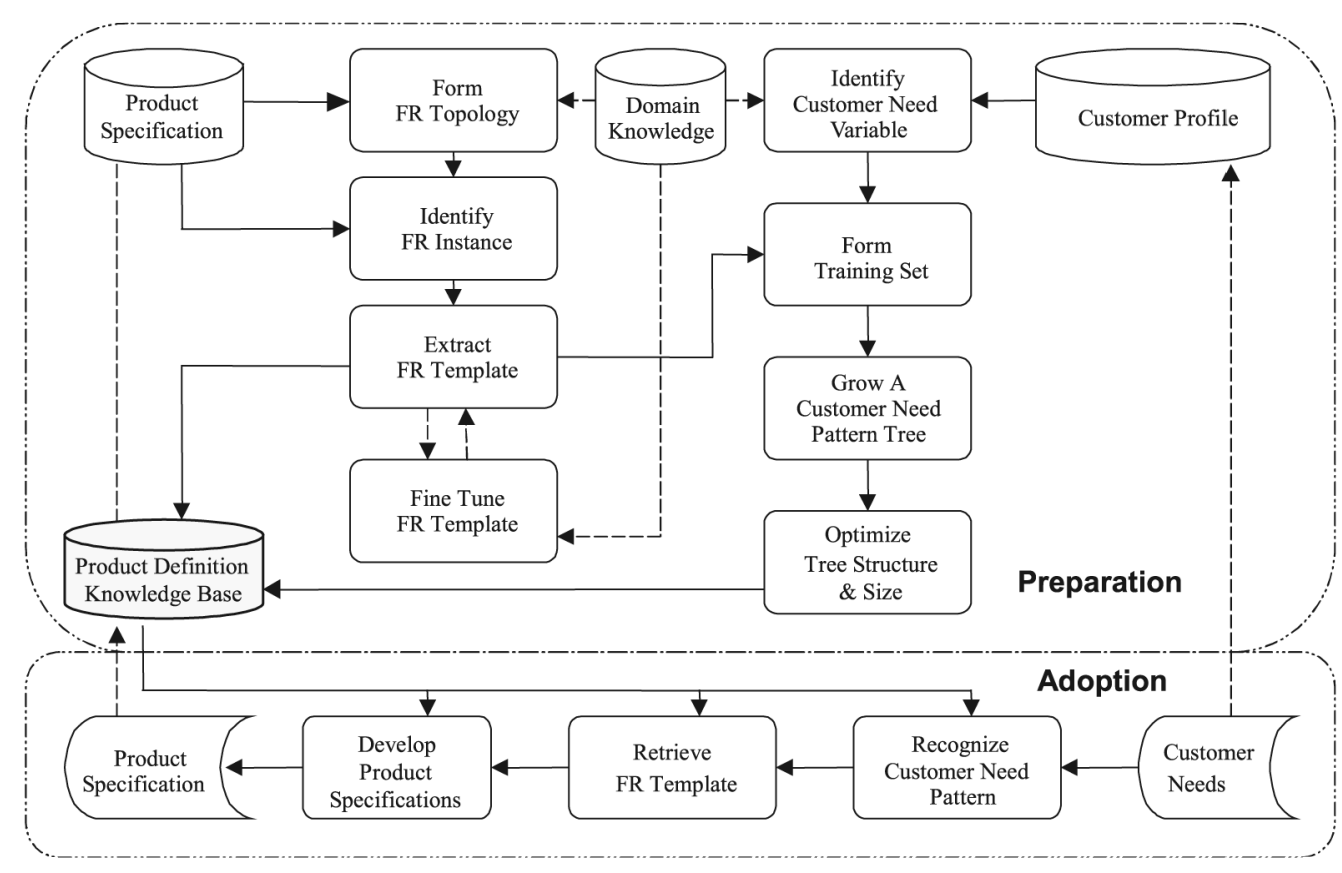


Xuehong Du, Jianxin Jiao and

Mitchell M. Tseng

Identifying customer need

patterns for customization and

personalization

Integrated Manufacturing

Systems

14/5 [2003] 387-396
Entity relationships involved in $\mathrm{CN}$ pattern facilitated product definition

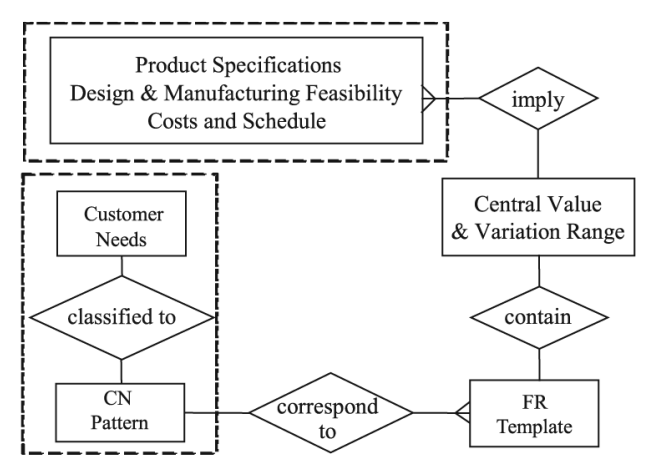

engineers with the knowledge necessary for defining a custom product. Such knowledge also enables them, and even the customer, to compare various tradeoffs and derive a comprehensive product definition within a short time frame. These heuristics are usually built into the product definition knowledge base. It is important to emphasize here that direct mapping based on $\mathrm{CN}$ patterns to develop a product specification does not mean to take over the engineers' creative work. Domain expertise is important to formulate $\mathrm{CN}$ patterns and explicate mapping relationships from $\mathrm{CN}$ patterns to FR templates.

\section{Application}

To test the feasibility of the proposed approach, we have applied it to an electronic product design - power supplies. Six product families of DC/DC converters are selected. Following the method in Tseng and Jiao (1998), the FR template of each product family is formulated separately. Each of them contains 151 parameters.

Four CNVs are identified as the determinant variables to identify $\mathrm{CN}$ patterns. The levels of each variable are set according to the existing company's offerings. Based on company documentation, ordering data of 54 products from the six families are used for analysis, which produces 226 valid samples. Half of them are used to construct the tree and the other half are used for validation purpose. The prediction rate of the $\mathrm{CN}$ tree is 91 per cent.

Development of a particular product specification means determining a whole set of the parameters. In fact, the six categories of most commonly requested customization only affect a subset of these parameters. In addition, some of the parameters are fixed because of the share of the same technology platform. The template can explicate what are variable parameters and how they vary in response to customization and personalization. This facilitates design engineers to narrow down their consideration set and to derive a feasible solution quickly.

Based on the result of the above experiment, a Web-based prototype system is developed for a noted manufacturer in this industry. It consists of an HTML form interface and a CGI-Win (VB) backend. The goal is to take advantage of the advanced information technology to reorganize and present the company's capability, and to provide good customer service by quick product definition and order processing. The screen shot of the system shown in Figure 6 illustrates how customers are guided to input their needs, where the FR topology is used to present customers with informed choices. Such a support to customization and personalization is implemented through underlying CR patterns, which organize customer preferences by product families. Figure 7 shows how the FR template is retrieved and used for designers to define product specifications. The Web-based user interface allows marketing personnel, customers and designers to access and browse the company's offerings more easily. In this sense, the proposed methodology provides useful guidelines for developing backend engines for e-commerce applications.

\section{Conclusions}

Accurate product definition and quick response to customization and personalization are premier competitive advantages for a manufacturer to maintain its market share and to excel itself among competitors. With clarifications of the distinction and relationship between customer needs and product specifications, this paper proposes an approach to effective product definition by identifying customer need patterns. While customer need patterns characterize customer preferences and are formulated from the customer perspective, functional requirement templates reflect the company's capability and are constructed from the engineering perspective. Their interrelationships are modeled using a customer need classification tree derived from previous design repositories using inductive learning techniques. The proposed approach not only facilitates manufacturers to achieve responsive product definition in routine businesses where customization and 
Xuehong Du, Jianxin Jiao and Mitchell M. Tseng

Identifying customer need patterns for customization and personalization

Integrated Manufacturing Systems

14/5 [2003] 387-396

\section{Figure 6}

Elicit customer needs based on the FR topology

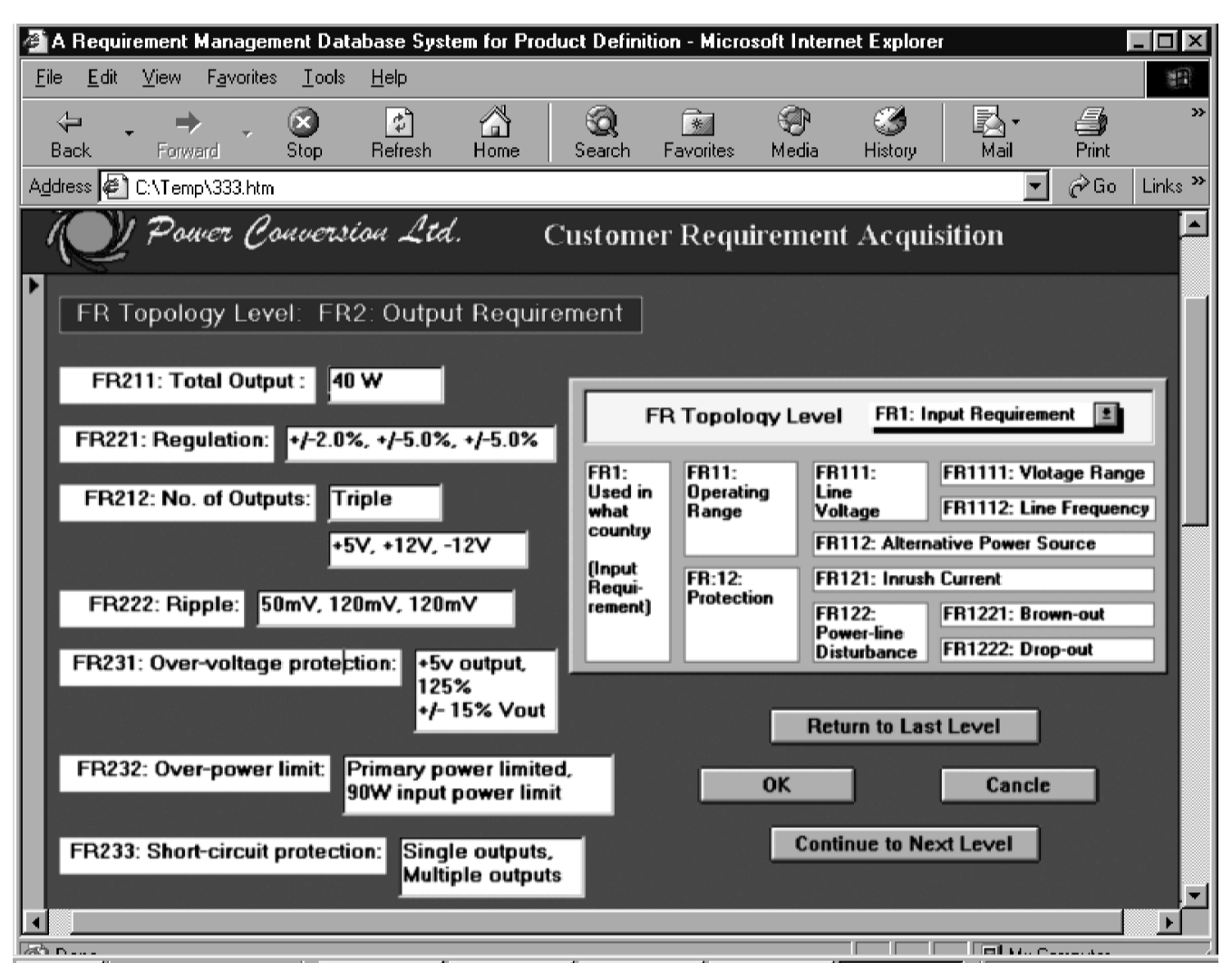

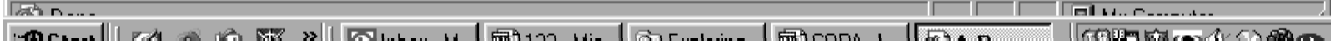

\section{Figure 7}

Define product specifications by instantiating the FR template

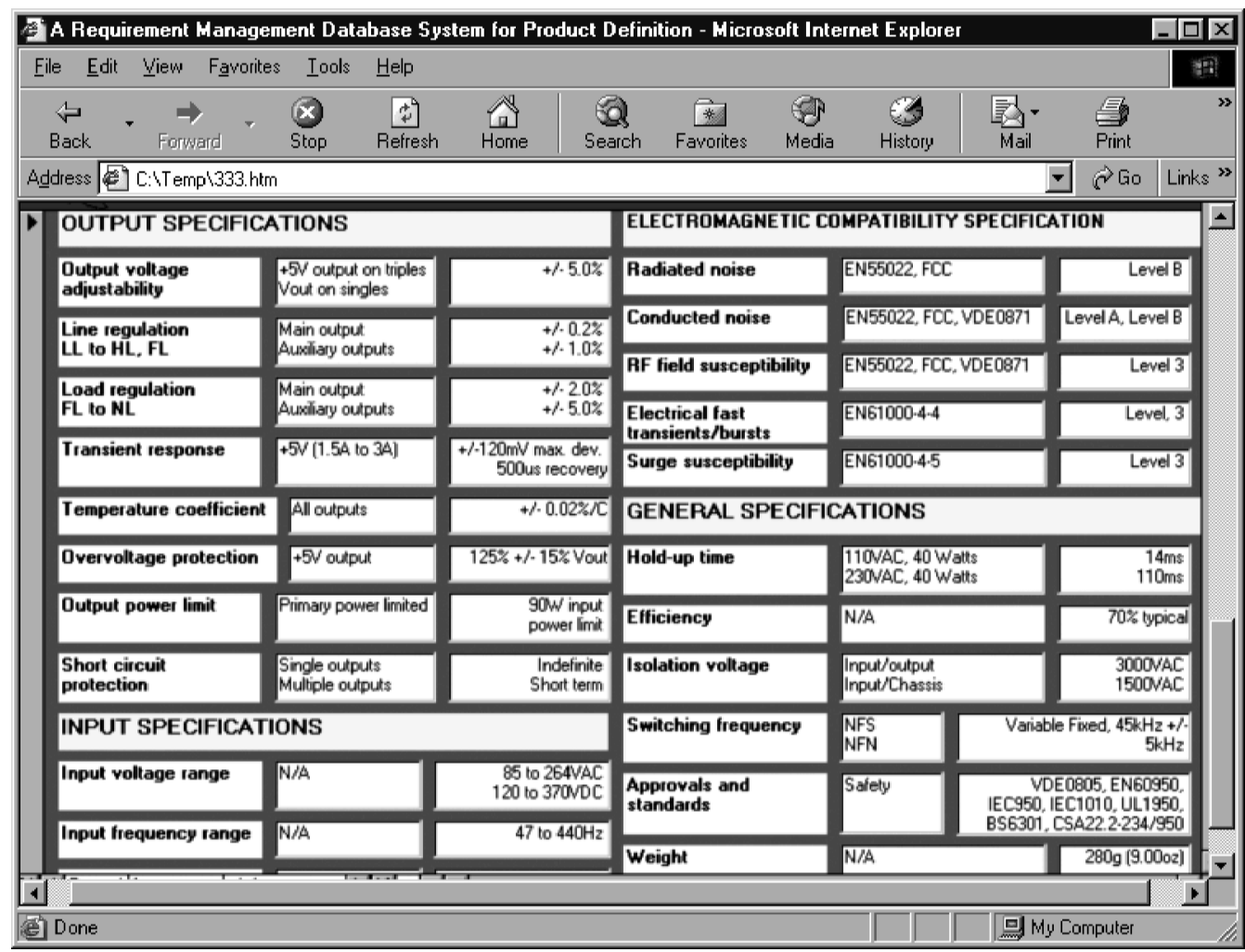


Xuehong Du, Jianxin Jiao and Mitchell M. Tseng

Identifying customer need patterns for customization and personalization

Integrated Manufacturing Systems

14/5 [2003] 387-396 personalization are frequently required, but also helps to discover customers' latent needs and increase the congruence of the company's capabilities with markets, as well as maintain the continuity of technology platforms and capital investment.

As an incipient endeavor, this research tackles product definition through customer need patterns. Some issues are worthy of further study:

- defining the typical and optimal structures to represent customer need patterns;

- how to integrate customer need classification with FR template formulation so as to better plan product offerings for customization and personalization;

- an expert system with learning capability and inference mechanisms seems imperative in practice; and

- under the framework of design domains, the proposed method has the potential to extend to subsequent domains, for example the design physical and process domains, so as to synchronize both product variety and process variety in implementing mass customization and personalization.

\section{References}

Anderson, D.M. (1997), Agile Product Development for Mass Customization, Irwin Professional Publishers, Chicago, IL.

Breiman, L. (1984), Classification and Regression Trees, Pacific Grove, Wadsworth \& Brooks/Cole Advanced Books \& Software, CA.

Churchill, G.A. (1995), Marketing Research: Methodological Foundations, 6th ed., Dryden Press, Forth Worth, TX.

Currim, I.S., Meyer, R.J. and Le, N.T. (1988), "Disaggregate tree-structured modeling of consumer choice data", Journal of Marketing Research, Vol. 25 No. 3, pp. 253-65.

$\mathrm{Du}, \mathrm{X} .$, Jiao, J. and Tseng, M.M. (2001), "Architecture of product family: fundamentals and methodology", Concurrent Engineering: Research and Application, in press.

The Economist (2000), "Business: all yours", Vol. 355 No. 8164, pp. 57-8.
The Economist (2001), "Business special: a long march mass customization”, Vol. 360 No. 8230, pp. 63-5.

Gunter, B. (1998), "Tree-based classification and regression”, Quality Progress, pp. 121-3.

Huffman, C. and Kahn, B.E. (1998), "Variety for sale: mass customization or mass confusion", Journal of Retailing, Vol. 74 No. 4, pp. 491-513.

Hutt, M.D. and Thomas, W.S. (1998), Business Marketing Management: A Strategic View of Industrial and Organizational Markets, 6th ed., Dryden Press, Fort Worth, TX.

Mayer, M.H. and Lehnerd, A.P. (1997), The Power of Product Platforms: Building Value and Cost Leadership, Free Press, New York, NY.

Morris, L.J. and Stauffer, L.A. (1994), “A design taxonomy for eliciting customer requirements", Computers and Industrial Engineering, Vol. 27 No. 1-4, pp. 557-60.

Peppers, D., Rogers, M. and Dorf, R. (1999), The One to One Fieldbook: The Complete Toolkit for Implementing a 1 to 1 Marketing Program, Currency/Doubleday, New York, NY

Pine, B.J. and Gilmore, J.H. (1999), The Experience Economy: Work is Theatre and Every Business a Stage, Harvard Business School Press, Boston, MA.

Pugh, S. (1991), Total Design: Integrated Methods for Successful Product Engineering, Addison-Wesley, Wokingham, MA.

Shannon, C.E. (1948), "A mathematical theory of communication”, Bell System Technology Journal, Vol. 27, July, pp. 379-423.

Suh, N.P. (1990), The Principles of Design, Oxford University Press, New York, NY.

Tseng, M.M. and Du, X. (1998), "Design by customers for mass customization products", CIRP Annals, Vol. 47 No. 1, pp. 103-6.

Tseng, M.M. and Jiao, J. (1996), "Design for mass customization”, CIRP Annals, Vol. 45 No. 1 , pp. 153-6.

Tseng, M.M. and Jiao, J. (1998), "Computer-aided requirement management for product definition: a methodology and implementation", Concurrent Engineering Research and Application, Vol. 6 No. 2, pp. 145-60.

Von Hippel, E. (1998), "Economics of product development by ysers: the impact of 'sticky' local information”, Management Science, Vol. 44 No. 5, pp. 629-44.

Zimmermann, H.J. (1987), Fuzzy Sets, Decision Making and Expert Systems, Kluwer Academic Publishers, Boston, MA. 